\title{
An Analysis of Record Statistics based on an Exponentiated Gumbel Model
}

\author{
Suk Bok Kang ${ }^{a}$, Jung In Seo ${ }^{a}$, Yongku Kim ${ }^{1, b}$ \\ ${ }^{a}$ Department of Statistics, Yeungnam University \\ ${ }^{b}$ Department of Statistics, Kyungpook National University
}

\begin{abstract}
This paper develops a maximum profile likelihood estimator of unknown parameters of the exponentiated Gumbel distribution based on upper record values. We propose an approximate maximum profile likelihood estimator for a scale parameter. In addition, we derive Bayes estimators of unknown parameters of the exponentiated Gumbel distribution using Lindley's approximation under symmetric and asymmetric loss functions. We assess the validity of the proposed method by using real data and compare these estimators based on estimated risk through a Monte Carlo simulation.
\end{abstract}

Keywords: Approximate maximum likelihood estimator, Bayesian estimation, exponentiated Gumbel distribution, record values.

\section{Introduction}

The Gumbel distribution is useful to predict maximum flood and rainfall levels as well as for climate modeling distribution. Nadarajah (2006) introduces the exponentiated Gumbel distribution as a generalization of the Gumbel distribution. The exponentiated Gumbel distribution is a Gumbel distribution if $\lambda=1$.

The probability density function (pdf) and cumulative distribution function (cdf) of the random variable $\mathrm{X}$ having an exponentiated Gumbel distribution are given by

$$
f(x)=\frac{\lambda}{\sigma}\left[1-\exp \left(-\exp \left(-\frac{x}{\sigma}\right)\right)\right]^{\lambda-1} \exp \left(-\frac{x}{\sigma}\right) \exp \left(-\exp \left(-\frac{x}{\sigma}\right)\right)
$$

and

$$
F(x)=1-\left[1-\exp \left(-\exp \left(-\frac{x}{\sigma}\right)\right)\right]^{\lambda}, \quad-\infty<x<\infty, \alpha>0, \sigma>0,
$$

where $\lambda$ is the shape parameter and $\sigma$ is the scale parameter. Note that the pdf (1.1) can be expressed as

$$
f(x)=\frac{1}{\sigma} \sum_{l=0}^{\infty} \frac{(-1)^{l} \Gamma(\lambda)}{l ! \Gamma(\lambda-l)} \exp \left(-\frac{x}{\sigma}\right) \exp \left(-(l+1) \exp \left(-\frac{x}{\sigma}\right)\right)
$$

\footnotetext{
${ }^{1}$ Corresponding author: Assistant professor, Department of Statistics, Kyungpook National University, Daegu, 702-701, Korea. E-mail: kim.1252@knu.ac.kr
} 
by using the power series expansion

$$
(1+u)^{\lambda}=\sum_{j=0}^{\infty}\left(\begin{array}{c}
\lambda \\
j
\end{array}\right) u^{j}=\sum_{j=0}^{\infty} \frac{\Gamma(\lambda+1)}{\Gamma(\lambda-j+1)} \frac{u^{j}}{j !} .
$$

The squared error loss function (SELF) is a symmetric loss function that assigns equal losses to overestimation and underestimation. Therefore, under the SELF, a Bayes estimator is defined by the posterior expectation. However, such a restriction may not be practical because overestimation is usually more serious than underestimation in the estimation of reliability and failure rate functions. In this case, the use of a symmetrical loss function may not be appropriate. To address this limitation, we consider an asymmetric loss function known as the LINEX loss function (LLF). The LLF, introduced by Varian (1975), has received considerable attention because of Zellner (1986).

The LLF may be expressed as $L(\Delta) \propto \exp (c \Delta)-c \Delta-1, c \neq 0$, where $\Delta=\hat{\theta}-\theta$. The sign and magnitude of the shape parameter $c$ represents the direction and degree of symmetry, respectively. If $c$ is positive, then overestimation is more serious than underestimation; however, the opposite is true if $\mathrm{c}$ is negative. For $c=1$, the LLF is quite asymmetric about zero, with overestimation being more costly than underestimation. If $c$ close to zero, then the LINEX loss is approximately the squared error loss and thus almost symmetric. Zellner (1986) expresses a Bayes estimator of $\theta$ under the LLF as $\hat{\theta}_{L}=-(1 / c) \log \left[E_{\pi}\left(e^{-c \theta}\right)\right]$, provided that there exists a finite expectation. Using these loss functions, Kim et al. (2011a) suggest a Bayes estimator for an exponentiated half-triangle distribution based on Type I censoring. Kim et al. (2011b) derive Bayes estimators of shape parameters, reliability functions, and failure rate functions for a family of exponentiated distributions based on Type II right censoring. Chandler (1952) is the first to examine record values and documented a number of basic properties of records. Record values arise in many real-life situations that involve weather, sports, and economics. A record model is closely related to models of order statistics; in addition, both appear in many statistical applications that are widely used in statistical modeling and inferences because they can be viewed as order statistics from a sample whose size is determined by the value and order of the occurrence of observations. In particular, Balakrishnan et al. (1992) established some recurrence relationships for single and double moments of lower record values from the Gumbel distribution. Jaheen (2003) derived Bayes estimators of unknown parameters of the Gompertz distribution based on upper record values and obtained prediction bounds for future upper record values. Soliman et al. (2006) obtained Bayes estimators based on record statistics for two unknown parameters of the Weibull distribution. Ahmadi and Balakrishnan (2011) have recently discussed the prediction of future order statistics based on the largest and smallest observations at the time of a new record.

Let $X_{1}, X_{2}, X_{3}, \ldots$ be a sequence of independent and identically distributed (iid) random variables with the cdf $F(x)$ and the pdf $f(x)$. Setting $Y_{n}=\max \left(X_{1}, X_{2}, \ldots, X_{n}\right), n \geq 1$, we say that $X_{j}$ is an upper record and denoted by $X_{U(j)}$ if $Y_{j}>Y_{j-1}, j>1$. The indices for which upper record values occur are given by the record times $\{U(n), n \geq 1\}$, where $U(n)=\max \left\{j \mid j>U(n-1), X_{j}>X_{U(n-1)}\right\}, n>1$, with $U(1)=1$. Subsequently, we denote a sequence of upper record values $x_{U(1)}, x_{U(2)}, \ldots, x_{U(n)}$ by $x_{1}, x_{2}, \ldots, x_{n}$ for simplicity.

\section{Maximum Likelihood Estimation}

In this section, we discuss a maximum profile likelihood estimator of the scale parameter $\sigma$ when data are upper record values. We begin by deriving a maximum likelihood estimator (MLE) of unknown parameters. Let $x_{1}, x_{2}, \ldots, x_{n}$ be a sequence of upper record values from an exponentiated Gumbel 
distribution. Then a likelihood function based on upper record values, $x_{1}, x_{2}, \ldots, x_{n}$, is then given by

$$
L(\sigma, \alpha)=f\left(x_{n}\right) \prod_{i=1}^{n-1} \frac{f\left(x_{i}\right)}{1-F\left(x_{i}\right)} .
$$

It follows, from (1.1), (1.2) and (2.1), that

$$
L(\sigma, \alpha)=\left(\frac{\lambda}{\sigma}\right)^{n}\left[1-\exp \left(-\exp \left(-\frac{x_{n}}{\sigma}\right)\right)\right]^{\lambda} \prod_{i=1}^{n} \frac{\exp \left(-\frac{x_{i}}{\sigma}\right) \exp \left(-\exp \left(-\frac{x_{i}}{\sigma}\right)\right)}{1-\exp \left(-\exp \left(-\frac{x_{i}}{\sigma}\right)\right)} .
$$

The natural logarithm of the likelihood function (2.2) is given by

$$
\begin{aligned}
\log L(\sigma, \lambda)= & n \log \lambda-n \log \sigma+\lambda \log \left[1-\exp \left(-\exp \left(-\frac{x_{n}}{\sigma}\right)\right)\right] \\
& -\sum_{i=1}^{n} \log \left[1-\exp \left(-\exp \left(-\frac{x_{i}}{\sigma}\right)\right)\right]-\sum_{i=1}^{n}\left(\frac{x_{i}}{\sigma}+\exp \left(\frac{x_{i}}{\sigma}\right)\right) .
\end{aligned}
$$

From (2.3), we have the likelihood equations for $\sigma$ and $\lambda$ as

$$
\begin{aligned}
\frac{\partial}{\partial \sigma} \log L(\sigma, \lambda) & =-\frac{n}{\sigma}+\lambda T_{1}\left(\sigma ; x_{n}\right)+\sum_{i=1}^{n}\left[T_{2}\left(\sigma ; x_{i}\right)-T_{1}\left(\sigma ; x_{i}\right)\right] \\
& =0
\end{aligned}
$$

and

$$
\begin{aligned}
\frac{\partial}{\partial \lambda} \log L(\sigma, \lambda) & =\frac{n}{\lambda}-T_{3}\left(\sigma ; x_{n}\right) \\
& =0
\end{aligned}
$$

where

$$
\begin{aligned}
& T_{1}\left(\sigma ; x_{n}\right)=\frac{\exp \left(-\frac{x}{\sigma}\right) \exp \left(-\exp \left(-\frac{x_{n}}{\sigma}\right)\right)}{1-\exp \left(-\exp \left(-\frac{x_{n}}{\sigma}\right)\right)} \frac{x_{n}}{\sigma^{2}}, \\
& T_{2}\left(\sigma ; x_{i}\right)=\left(1-\exp \left(-\frac{x_{i}}{\sigma}\right)\right) \frac{x_{i}}{\sigma^{2}} \\
& T_{3}\left(\sigma ; x_{n}\right)=-\log \left[1-\exp \left(-\exp \left(-\frac{x_{n}}{\sigma}\right)\right)\right] .
\end{aligned}
$$

If the scale parameter $\sigma$ is known, then the MLE of the shape parameter $\lambda$, denote by $\hat{\lambda}$, can be obtained as

$$
\hat{\lambda}(\sigma)=\frac{n}{T_{3}\left(\sigma ; x_{n}\right)} .
$$

Using the MLE $\hat{\lambda}(\sigma)$ in (2.6), we can express the profile likelihood function of the scale parameter $\sigma$ as $L_{p}(\sigma)=L(\sigma, \hat{\lambda}(\sigma))$. Thus, the profile likelihood equation for $\sigma$ is given by

$$
\begin{aligned}
\frac{\partial}{\partial \sigma} \log L_{p}(\sigma) & =-\frac{n}{\sigma}+n \frac{T_{1}\left(\sigma ; x_{n}\right)}{T_{3}\left(\sigma ; x_{n}\right)}+\sum_{i=1}^{n}\left[T_{2}\left(\sigma ; x_{i}\right)-T_{1}\left(\sigma ; x_{i}\right)\right] \\
& =0
\end{aligned}
$$


We can find the profile MLE $\hat{\sigma}$ of $\sigma$ by solving the above equation (2.7). Unfortunately, this equation cannot be solved explicitly; therefore, we solve it by using a numerical method such as the Newton-Raphson method. The MLE of the shape parameter $\lambda$ is obtained by replacing $\sigma$ with $\hat{\sigma}$ in the equation (2.6).

\section{Approximate Maximum Likelihood Estimation}

As discussed earlier, because equation (2.7) is very complicated, it does not admit an explicit solution for $\sigma$. Therefore, we will derive the approximate MLE (AMLE) of $\sigma$ by using the approximate profile likelihood equation. A number of studies have considered the AMLE (see Kang et al., 2009; Kang and Seo, 2011).

Let $Z_{i}=X_{i} / \sigma$. Then it has a standard exponentiated Gumbel distribution with the pdf and the cdf

$$
f\left(z_{i}\right)=\lambda\left[1-\exp \left(-\exp \left(-z_{i}\right)\right)\right]^{\lambda-1} \exp \left(z_{i}\right) \exp \left(-\exp \left(-z_{i}\right)\right)
$$

and

$$
F\left(z_{i}\right)=1-\left[1-\exp \left(-\exp \left(-z_{i}\right)\right)\right]^{\lambda}, \quad-\infty<z<\infty, \lambda>0,
$$

respectively. The profile likelihood equation (2.7) can be written as

$$
\begin{aligned}
\frac{\partial}{\partial \sigma} L_{p}(\sigma) & =-\frac{1}{\sigma}\left[n+n \frac{G_{1}\left(z_{n}\right)}{G_{3}\left(z_{n}\right)} z_{n}+\sum_{i=1}^{n} G_{2}\left(z_{i}\right) z_{i}+\sum_{i=1}^{n} G_{1}\left(z_{i}\right) z_{i}\right] \\
& =0,
\end{aligned}
$$

where

$$
\begin{aligned}
G_{1}\left(z_{i}\right) & =\frac{\exp \left(-z_{i}\right) \exp \left(-\exp \left(-z_{i}\right)\right)}{1-\exp \left(-\exp \left(-z_{i}\right)\right)}, \\
G_{2}\left(z_{i}\right) & =\exp \left(-z_{i}\right)-1, \\
G_{3}\left(z_{n}\right) & =\log \left[1-\exp \left(-\exp \left(-z_{n}\right)\right)\right], \\
\xi_{i} & =F^{-1}\left(U_{i}\right)=-\log \left[-\log \left(1-\left(1-U_{i}\right)^{\frac{1}{\lambda}}\right)\right],
\end{aligned}
$$

where $U_{i}$ is a uniformly distributed random variate.

Using the Taylor series, we approximate the following functions:

$$
\begin{aligned}
\frac{G_{1}\left(z_{n}\right)}{G_{3}\left(z_{n}\right)} & \approx \alpha_{1 n}+\beta_{1 n} z_{n}, \\
G_{2}\left(z_{i}\right) & \approx \gamma_{1 i}+\eta_{1 i} z_{i}, \\
G_{1}\left(z_{i}\right) & \approx \kappa_{1 i}+\delta_{1 i} z_{i},
\end{aligned}
$$

where

$$
\begin{aligned}
\alpha_{1 n} & =\frac{G_{1}\left(\xi_{n}\right)}{G_{3}\left(\xi_{n}\right)}-\left[\frac{G_{1}\left(\xi_{n}\right)}{G_{3}\left(\xi_{n}\right)}\left(\frac{G_{1}\left(\xi_{n}\right)}{G_{3}\left(\xi_{n}\right)}+G_{2}\left(\xi_{n}\right)+G_{1}\left(\xi_{n}\right)\right)\right] \xi_{n}, \\
\beta_{1 n} & =\left[\frac{G_{1}\left(\xi_{n}\right)}{G_{3}\left(\xi_{n}\right)}\left(\frac{G_{1}\left(\xi_{n}\right)}{G_{3}\left(\xi_{n}\right)}+G_{2}\left(\xi_{n}\right)+G_{1}\left(\xi_{n}\right)\right)\right], \\
\gamma_{1 i} & =G_{2}\left(\xi_{i}\right)+\exp \left(\xi_{i}\right) \xi_{i}, \quad \eta_{1 i}=-\exp \left(-\xi_{i}\right), \\
\kappa_{1 i} & =G_{1}\left(\xi_{i}\right)\left[1-\left(G_{2}\left(\xi_{i}\right)+G_{1}\left(\xi_{i}\right)\right) \xi_{i}\right], \quad \delta_{1 i}=G_{1}\left(\xi_{i}\right)\left[G_{2}\left(\xi_{i}\right)+G_{1}\left(\xi_{i}\right)\right] .
\end{aligned}
$$


Entering equations (3.4), (3.5), and (3.6) into the equation (3.3), we obtain the following approximate profile likelihood equation:

$$
\begin{aligned}
\frac{\partial}{\partial \sigma} L_{p}(\sigma) & \simeq-\frac{1}{\sigma}\left[n+n\left(\alpha_{1 n}+\beta_{1 n} z_{n}\right) z_{n}+\sum_{i=1}^{n}\left(\gamma_{1 i}+\eta_{1 i} z_{i}\right) z_{i}+\sum_{i=1}^{n}\left(\kappa_{1 i}+\delta_{1 i}\right) z_{i}\right] \\
& =0 .
\end{aligned}
$$

After solving the quadratic equation (3.7) for $\sigma$, we obtain the AMLE of $\sigma$ as

$$
\tilde{\sigma}_{1}=\frac{-A+\sqrt{A^{2}-4 n B}}{2 n},
$$

where

$$
\begin{aligned}
& A=n x_{n} \alpha_{1 n}+\sum_{i=1}^{n} x_{i}\left(\kappa_{1 i}+\gamma_{1 i}\right), \\
& B=n x_{n}^{2} \beta_{1 n}+\sum_{i=1}^{n} x_{i}^{2}\left(\delta_{1 i}+\eta_{1 i}\right) .
\end{aligned}
$$

In addition, we obtain the AMLE of the shape parameter $\lambda$, denoted by $\tilde{\lambda}_{1}$, by replacing $\sigma$ with $\tilde{\sigma}_{1}$ in equation (2.6).

Similarly, we approximate the other functions as follows:

$$
\begin{aligned}
\frac{G_{1}\left(z_{n}\right)}{G_{3}\left(z_{n}\right)} z_{n} & \approx \alpha_{2 n}+\beta_{2 n} z_{n}, \\
G_{2}\left(z_{i}\right) z_{i} & \approx \gamma_{2 i}+\eta_{2 i} z_{i}, \\
G_{1}\left(z_{i}\right) z_{i} & \approx \kappa_{2 i}+\delta_{2 i} z_{i},
\end{aligned}
$$

where

$$
\begin{aligned}
\alpha_{2 n} & =-\frac{G_{1}\left(\xi_{n}\right)}{G_{3}\left(\xi_{n}\right)}\left[\frac{G_{1}\left(\xi_{n}\right)}{G_{3}\left(\xi_{n}\right)} \xi_{n}+G_{2}\left(\xi_{n}\right)+G_{1}\left(\xi_{n}\right)\right] \xi_{n}, \\
\beta_{2 n} & =\frac{G_{1}\left(\xi_{n}\right)}{G_{3}\left(\xi_{n}\right)}\left[\frac{G_{1}\left(\xi_{n}\right)}{G_{3}\left(\xi_{n}\right)} \xi_{n}+G_{2}\left(\xi_{n}\right)+G_{1}\left(\xi_{n}\right)+1\right], \\
\gamma_{2 i} & =\exp \left(-\xi_{i}\right) \xi_{i}, \quad \eta_{2 i}=G_{2}\left(\xi_{i}\right)-\exp \left(-\xi_{i}\right) \xi_{i}, \\
\kappa_{2 i} & =-G_{1}\left(\xi_{i}\right)\left[G_{2}\left(\xi_{i}\right)+G_{1}\left(\xi_{i}\right)\right] \xi_{i}^{2}, \quad \delta_{2 i}=G_{1}\left(\xi_{i}\right)\left[\left(G_{2}\left(\xi_{i}\right)+G_{1}\left(\xi_{i}\right)\right) \xi_{i}+1\right] .
\end{aligned}
$$

By using equations (3.9), (3.10), and (3.11), we obtain the following approximate profile likelihood equation:

$$
\begin{aligned}
\frac{\partial}{\partial \sigma} L_{p}(\sigma) & \simeq-\frac{1}{\sigma}\left[n+n\left(\alpha_{2 n}+\beta_{2 n} z_{n}\right)+\sum_{i=1}^{n}\left(\gamma_{2 i}+\eta_{2 i} z_{i}\right)+\sum_{i=1}^{n}\left(\kappa_{2 i}+\delta_{2 i} z_{i}\right)\right] \\
& =0 .
\end{aligned}
$$

By solving equation (3.12) for $\sigma$, we obtain another AMLE of $\sigma$ :

$$
\tilde{\sigma}_{2}=-\frac{C}{n+D}
$$


where

$$
\begin{aligned}
& C=n x_{n} \beta_{2 n}+\sum_{i=1}^{n} x_{i}\left(\delta_{2 i}+\eta_{2 i}\right), \\
& D=n \alpha_{2 n}+\sum_{i=1}^{n}\left(\kappa_{2 i}+\gamma_{2 i}\right) .
\end{aligned}
$$

As in the case of the AMLE $\tilde{\lambda}_{1}$, we obtain another AMLE of $\lambda$, denoted by $\tilde{\lambda}_{2}$, by replacing $\sigma$ with $\tilde{\sigma}_{2}$ in equation (2.6). Unlike the MLE $\hat{\sigma}$, it need not to use iterative methods because AMLEs $\tilde{\sigma}_{1}$ and $\tilde{\sigma}_{2}$ can be solved explicitly. Note that obtained AMLEs can be used as initial values for iteration and the estimator $\tilde{\sigma}_{2}$ is simpler than the estimator $\tilde{\sigma}_{1}$ because $\tilde{\sigma}_{2}$ is a linear combination of available order statistics.

\section{Bayes Estimation}

In this section, we derive Bayes estimators of unknown parameters of the exponentiated Gumbel distribution based on upper record values. Here the loss functions are the SELF (symmetric) and the LLF (asymmetric).

\subsection{Prior and posterior distributions}

Suppose that $\lambda$ conditional upon $\sigma$ has a $\operatorname{gamma}(v, \delta / \sigma)$ distribution with the pdf

$$
\pi_{1}(\lambda \mid \sigma)=\frac{\delta^{v}}{\Gamma(v) \sigma^{v}} \lambda^{v-1} \exp \left(-\frac{\lambda \delta}{\sigma}\right), \quad \lambda>0, \quad(\delta>0, v>0),
$$

which is a natural conjugate prior. It follows from (2.2) and (4.1) that the conditional posterior of $\lambda \mid \sigma$ is given by

$$
\pi_{1}^{*}(\lambda \mid \sigma)=\frac{B_{2}^{n+v}}{\Gamma(n+v)} \lambda^{n+v-1} \exp \left(-\lambda B_{2}\right), \quad \lambda>0, \quad(\delta>0, v>0)
$$

where

$$
B_{2}\left(\sigma ; x_{n}\right)=\frac{\delta}{\sigma}+T_{3}\left(\sigma ; x_{n}\right)
$$

Note that $\pi_{1}^{*}(\lambda \mid \sigma)$ is a $\operatorname{Gamma}\left(n+v, B_{2}\right)$. If $v=\delta=0$, then the Bayes estimator of $\lambda$ under the SELF is identical to the MLE $\hat{\lambda}$ because the Bayes estimator under the SELF is the posterior mean. Further, we suppose that the scale parameter $\sigma$ has an inverted gamma prior,

$$
\pi_{2}(\sigma)=\frac{b^{a}}{\Gamma(a)} \frac{1}{\sigma^{a+1}} \exp \left(-\frac{b}{\sigma}\right), \quad \sigma>0, \quad(a>0, b>0) .
$$

Here we consider the joint prior distribution as the product of the conditional prior of $\lambda$ for given $\sigma$ and the inverted gamma prior for $\sigma$. That is, the joint posterior distribution function of $\lambda$ and $\sigma$ can be written as

$$
\begin{aligned}
\pi^{*}(\sigma, \lambda) & =L(\sigma, \lambda) \pi(\sigma, \lambda) / \int_{\sigma} \int_{\lambda} L(\sigma, \lambda) \pi(\sigma, \lambda) \mathrm{d} \sigma \mathrm{d} \lambda \\
& =\frac{G}{\sigma^{n+a+v+1}} \lambda^{n+v-1} B_{1}\left(\sigma ; x_{i}\right) \exp \left(-\lambda B_{2}\left(\sigma ; x_{n}\right)\right),
\end{aligned}
$$


where

$$
B_{1}\left(\sigma ; x_{i}\right)=\sigma^{2} \exp \left(-\frac{b}{\sigma}\right) \prod_{i=1}^{n} \frac{T_{1}\left(\sigma ; x_{i}\right)}{x_{i}}
$$

and $G$ is the normalizing constant given by

$$
G^{-1}=\Gamma(n+v) \int_{0}^{\infty} \frac{B_{1}\left(\sigma ; x_{i}\right)}{\sigma^{n+v+a+1}}\left(\frac{1}{B_{2}\left(\sigma ; x_{n}\right)}\right)^{n+v} \mathrm{~d} \sigma .
$$

\subsection{Lindley's approximation}

Let $H(\sigma, \lambda)$ is any function of $\sigma$ and $\lambda$,. Then Bayes estimators of $H(\sigma, \lambda)$ under the SELF and the LLF are

$$
\hat{H}_{S}(\sigma, \lambda)=\frac{\int_{\sigma} \int_{\lambda} H(\sigma, \lambda) L(\sigma, \lambda) \pi(\sigma, \lambda) \mathrm{d} \sigma \mathrm{d} \lambda}{\int_{\sigma} \int_{\lambda} L(\sigma, \lambda) \pi(\sigma, \lambda) \mathrm{d} \sigma \mathrm{d} \lambda}
$$

and

$$
\hat{H}_{L}(\sigma, \lambda)=-\frac{1}{c} \log \left(\frac{\int_{\lambda} \int_{\sigma} e^{-c H(\sigma, \lambda)} L(\sigma, \lambda) \pi(\sigma, \lambda) \mathrm{d} \sigma \mathrm{d} \lambda}{\int_{\lambda} \int_{\sigma} L(\sigma, \lambda) \pi(\sigma, \lambda) \mathrm{d} \sigma \mathrm{d} \lambda}\right)
$$

respectively.

If $H(\sigma, \lambda)=\sigma$, then we obtain the Bayes estimators $\hat{\sigma}_{S}$ and $\hat{\sigma}_{L}$, whereas if $H(\sigma, \lambda)=\lambda$, then we get $H_{S}(\sigma, \lambda)=\hat{\lambda}_{S}$ and $H_{L}(\sigma, \lambda)=\hat{\lambda}_{L}$, respectively. The ratios of integrals in (4.5) and (4.6) do not to take a closed form; therefore, we consider Lindley's approximation technique (Lindley, 1980) that technique has been widely used (see, for example, Howlader and Hossain, 2002; Kundu and Gupta, 2005; Soliman et al., 2006). Based on Lindley's approximation, we have the following Bayes estimators of $\sigma$ and $\lambda$ under the SELF:

$$
\hat{\sigma}_{S}=\hat{\sigma}+\frac{1}{2} \psi_{1}+\psi_{2}
$$

and

$$
\hat{\lambda}_{S}=\hat{\lambda}+\frac{1}{2} \psi_{3}+\psi_{4}
$$

In addition, the Bayes estimators of $\sigma$ and $\lambda$ under the LLF are

$$
\hat{\sigma}_{L}=\hat{\sigma}-\frac{1}{c} \log \left[1+\frac{c\left(c \tau_{11}-\psi_{1}-2 \psi_{2}\right)}{2}\right]
$$

and

$$
\hat{\lambda}_{L}=\hat{\lambda}-\frac{1}{c} \log \left[1+\frac{c\left(c \tau_{22}-\psi_{3}-2 \psi_{4}\right)}{2}\right]
$$


Table 1: The maximum monthly temperatures from 2011, in Seoul.

\begin{tabular}{|c|c|c|c|c|c|c|c|c|c|c|c|}
\hline Jan & Feb & Mar & Apr & May & Jun & Jul & Aug & Sep & Oct & Nov & Dec \\
\hline-3.6 & 6.2 & 10.7 & 13.5 & 24.4 & 26.6 & 28.7 & 28.9 & 27 & 17.3 & 19.9 & 5.8 \\
\hline
\end{tabular}

Table 2: The MLEs and AMLEs of $\sigma$ and $\lambda$ for real data.

\begin{tabular}{cccccc}
\hline \hline$\hat{\sigma}$ & $\tilde{\sigma}_{1}$ & $\tilde{\sigma}_{2}$ & $\hat{\lambda}$ & $\tilde{\lambda}_{1}$ & $\tilde{\lambda}_{2}$ \\
\hline 1.24074 & 1.33380 & 1.25165 & 0.34346 & 0.36922 & 0.34648 \\
\hline \hline
\end{tabular}

Table 3: The Bayes estimates of $\sigma$ and $\lambda$ for real data.

\begin{tabular}{cccccc}
\hline \hline$\hat{\sigma}_{S}$ & $\hat{\sigma}_{L}(c=-1)$ & $\hat{\sigma}_{L}(c=1)$ & $\hat{\lambda}_{S}$ & $\hat{\lambda}_{L}(c=-1)$ & $\hat{\lambda}_{L}(c=1)$ \\
\hline 1.23976 & 1.24050 & 1.23901 & 0.34243 & 0.34984 & 0.33504 \\
\hline \hline
\end{tabular}

where

$$
\begin{aligned}
& \psi_{1}=\tau_{11}^{2} L_{30}^{*}+\tau_{21} \tau_{22} L_{03}^{*}+3 \tau_{11} \tau_{12} L_{21}^{*}, \\
& \psi_{2}=p_{1} \tau_{11}+p_{2} \tau_{12}, \\
& \psi_{3}=\tau_{22}^{2} L_{03}^{*}+\tau_{12} \tau_{11} L_{30}^{*}+\left(\tau_{11} \tau_{22}+2 \tau_{12}^{2}\right) L_{21}^{*}, \\
& \psi_{4}=p_{1} \tau_{21}+p_{2} \tau_{22} .
\end{aligned}
$$

See the Appendix for the proof of the Bayes estimators.

\section{Illustrative Example and Simulation Study}

In this section, we present two examples to illustrate the previously discussed estimation methods.

\subsection{Real data}

The data given in Table 1 consist of maximum monthly temperatures from 2011, in Seoul.

From the above data, we observe 8 upper record values.

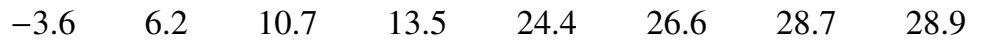

Using the formulae presented in Section 2, Section 3, and Section 4, we obtain the ML and Bayes estimates of the scale parameter $\sigma$ and the shape parameter $\lambda$. These values are given in Table 2 and Table 3. We see that all estimates have nearly the same values.

\subsection{Simulation result}

It is difficult to determine and compare MLEs and Bayes estimators using real data because we do not know the true values. Therefore, we conduct a simulation study to assess the efficiency ML and Bayes estimation methods in terms of the estimated risk. For this, we employ the following procedure to determine the estimated risk for Bayes estimators: We first set $E(\sigma)=1, E(\lambda)=1.5$, and $V(\sigma)=V(\lambda)=0.15$ from the prior distributions (4.1) and (4.3). We then obtain the hyperparameters $a$ and $b$ of the inverted gamma prior (4.3) by solving the prior information $E(\sigma)$ and $V(\sigma)$. Then, by solving the prior information $E(\lambda)$ and $V(\sigma)$ after replacing $\sigma$ with $E(\sigma)$ in the gamma prior (4.1), we obtain the hyperparameters $v$ and $\delta$ of the gamma prior (4.1). Note that $E(\sigma)=1$ and $E(\lambda)=1.5$ are the actual values for $\sigma$ and $\lambda$, respectively. We generate upper record values from the exponentiated Gumbel distribution with $\sigma=E(\sigma)$ and $\lambda=E(\lambda)$ to obtain the estimated risk for Bayes estimators. We calculate the estimated risks for each estimator as the average of their squared deviations for 
Table 4: The estimated risks of the estimators of $\sigma$ and $\lambda$.

\begin{tabular}{|c|c|c|c|c|c|c|}
\hline$n$ & $\hat{\sigma}$ & $\tilde{\sigma}_{1}$ & $\tilde{\sigma}_{2}$ & $\hat{\lambda}$ & $\tilde{\lambda}_{1}$ & $\tilde{\lambda}_{2}$ \\
\hline 6 & 0.10672 & 0.15210 & 0.18163 & 0.17591 & 0.21913 & 0.24536 \\
\hline 7 & 0.08096 & 0.12534 & 0.15966 & 0.11539 & 0.16052 & 0.19366 \\
\hline 8 & 0.06586 & 0.10904 & 0.14336 & 0.07070 & 0.11392 & 0.14807 \\
\hline 9 & 0.05561 & 0.09777 & 0.13080 & 0.03864 & 0.07674 & 0.10893 \\
\hline 10 & 0.04841 & 0.08941 & 0.12082 & 0.01800 & 0.04751 & 0.07587 \\
\hline$n$ & $\hat{\sigma}_{S}$ & $\hat{\sigma}_{L}(c=-1)$ & $\hat{\sigma}_{L}(c=1)$ & $\hat{\lambda}_{S}$ & $\hat{\lambda}_{L}(c=-1)$ & $\hat{\lambda}_{L}(c=1)$ \\
\hline 6 & 0.18192 & 0.18132 & 0.18256 & 0.12282 & 0.20375 & 0.04863 \\
\hline 7 & 0.13995 & 0.13918 & 0.14076 & 0.06518 & 0.12127 & 0.02301 \\
\hline 8 & 0.11614 & 0.11534 & 0.11696 & 0.03930 & 0.07640 & 0.01375 \\
\hline 9 & 0.09988 & 0.09911 & 0.10067 & 0.02529 & 0.04876 & 0.00879 \\
\hline 10 & 0.08830 & 0.08758 & 0.08904 & 0.01705 & 0.03111 & 0.00615 \\
\hline
\end{tabular}

10,000 repetitions:

$$
\frac{1}{n} \sum_{i=1}^{n}\left(\theta_{t}-\hat{\theta}\right)^{2}
$$

Here $\theta_{t}$ and $\hat{\theta}$ are the actual value and the estimate of $\theta$, respectively. Table 4 shows the estimated risks of $\sigma$ and $\lambda$. As expected, the estimated risk for all estimators decrease as the sample size $n$ increases. For the scale parameter $\sigma$, the MLE $\hat{\sigma}$ is more efficient than the AMLEs $\tilde{\sigma}_{1}$ and $\tilde{\sigma}_{2}$. However, the AMLE has a closed form. In addition, we see that the asymmetric Bayes estimator $\hat{\sigma}_{L}$ is superior to the symmetric Bayes estimator $\hat{\sigma}_{s}$ for $c=-1$. However, the symmetric Bayes estimator $\hat{\sigma}_{S}$ is superior to the asymmetric Bayes estimator $\hat{\sigma}_{L}$ for $c=1$. For the shape parameter $\lambda$, when the MLE is compared with the AMLE, we have the same result. In addition, the asymmetric Bayes estimator $\hat{\lambda}_{L}(c=1)$ has the lowest estimated risk among Bayes estimators. That is, MLEs outperform their AMLEs and Bayes counterparts for the scale parameter $\sigma$, but overall, Bayes estimators outperform their MLE and AMLE counterparts for the shape parameter $\lambda$, particularly for asymmetric loss functions such as LINEX, when $c=1$.

\section{Concluding Remarks}

This paper develops a maximum profile likelihood estimator of scale and shape parameters. Because the MLE cannot be solved explicitly, we propose the AMLE as an alternative estimator. We can obtain AMLEs by solving the approximate profile likelihood equation. In addition, we obtain approximate Bayes estimators of scale and shape parameters using Lindley's approximation method. We compare these estimators based on estimated risk. The results suggest that MLEs are superior to their AML and Bayes counterparts for scale parameters but that Bayes estimators outperform their ML and AML counterparts for shape parameters, particularly for asymmetric loss functions such as LINEX, when $c=1$. The results suggest that Bayes estimators are dependent on prior information and the shape parameter $c$ of the LINEX loss function; therefore, asymmetric Bayes estimators outperform other estimators if there are suitable $c$ values and prior information.

\section{Appendix:}

For the two-parameter $(\sigma, \lambda)$, the Lindley's approximation can be written as

$$
\hat{H}=H(\hat{\sigma}, \hat{\lambda})+\frac{1}{2}\left[A+L_{30}^{*} B_{12}+L_{03}^{*} B_{21}+L_{21}^{*} C_{12}+L_{12}^{*} C_{21}\right]+p_{1} A_{12}+p_{2} A_{21},
$$


where

$$
\begin{aligned}
A & =\sum_{i=1}^{2} \sum_{j=1}^{2} w_{i j} \tau_{i j}, \quad L_{i j}^{*}=\frac{\partial^{i+j} \log L(\sigma, \lambda)}{\partial \sigma^{i} \partial \lambda^{j}}, \quad i, j=0,1,2,3 \text { with } i+j=3, \\
p_{1} & =\frac{\partial p}{\partial \sigma}, \quad p_{2}=\frac{\partial p}{\partial \lambda}, \quad w_{1}=\frac{\partial H}{\partial \sigma}, \quad w_{2}=\frac{\partial H}{\partial \lambda}, \quad w_{i j}=\frac{\partial^{2} H}{\partial \sigma \partial \lambda}, \quad p=\log \pi(\sigma, \lambda), \\
A_{i j} & =w_{i} \tau_{i i}+w_{j} \tau_{j i}, \quad B_{i j}=\left(w_{i} \tau_{i i}+w_{j} \tau_{i j}\right) \tau_{i i}, \quad C_{i j}=3 w_{i} \tau_{i i} \tau_{i j}+w_{j}\left(\tau_{i i} \tau_{j j}+2 \tau_{i j}^{2}\right) .
\end{aligned}
$$

Here, the elements $\tau_{i j}$ can be obtained as

$$
\tau_{11}=\frac{V}{S V-U^{2}}, \quad \tau_{22}=\frac{S}{S V-U^{2}}, \quad \tau_{12}=\tau_{21}=-\frac{U}{S V-U^{2}} .
$$

where

$$
\begin{aligned}
S & =-\frac{\partial^{2}}{\partial \sigma^{2}} \log L(\sigma, \lambda)=-\frac{n}{\sigma^{2}}-\lambda T_{4}\left(\sigma ; x_{n}\right)-\sum_{i=1}^{n} T_{5}\left(\sigma ; x_{i}\right)+\sum_{i=1}^{n} T_{4}\left(\sigma ; x_{i}\right), \\
V & =-\frac{\partial^{2}}{\partial \lambda^{2}} \log L(\sigma, \lambda)=\frac{n}{\lambda^{2}}, \quad U=-\frac{\partial^{2}}{\partial \sigma \partial \lambda} \log L(\sigma, \lambda)=-T_{1}\left(\sigma ; x_{n}\right), \\
T_{4}\left(\sigma ; x_{i}\right) & =T_{1}\left(\sigma ; x_{i}\right)\left[T_{2}\left(\sigma ; x_{i}\right)-T_{1}\left(\sigma ; x_{i}\right)-\frac{2}{\sigma}\right], \\
T_{5}\left(\sigma ; x_{i}\right) & =T_{2}\left(\sigma ; x_{i}\right)\left(\frac{x_{i}}{\sigma^{2}}-\frac{2}{\sigma}\right)-\frac{x_{i}^{2}}{\sigma^{4}} .
\end{aligned}
$$

In addition, the values of $L_{i j}^{*}$ can be obtained as follows for $i, j=0,1,2,3$,

$$
\begin{aligned}
& L_{30}^{*}=-\frac{2 n}{\sigma^{3}}+\lambda T_{6}\left(\sigma ; x_{n}\right)+\sum_{i=1}^{n} T_{7}\left(\sigma ; x_{i}\right)-\sum_{i=1}^{n} T_{6}\left(\sigma ; x_{i}\right), \\
& L_{03}^{*}=\frac{2 n}{\lambda^{3}}, \quad L_{21}^{*}=T_{4}\left(\sigma ; x_{n}\right), \quad L_{12}^{*}=0
\end{aligned}
$$

where

$$
\begin{aligned}
& T_{6}\left(\sigma ; x_{i}\right)=T_{4}\left(\sigma ; x_{i}\right)\left[T_{2}\left(\sigma ; x_{i}\right)-T_{1}\left(\sigma ; x_{i}\right)-\frac{2}{\sigma}\right]+T_{1}\left(\sigma ; x_{i}\right)\left[T_{5}\left(\sigma ; x_{i}\right)-T_{4}\left(\sigma ; x_{i}\right)+\frac{2}{\sigma^{2}}\right] \\
& T_{7}\left(\sigma ; x_{i}\right)=T_{5}\left(\sigma ; x_{i}\right)\left(\frac{x_{i}}{\sigma^{2}}-\frac{2}{\sigma}\right)+T_{2}\left(\sigma ; x_{i}\right)\left(\frac{2}{\sigma^{2}}-\frac{2 x_{i}}{\sigma^{3}}\right)+\frac{4 x_{i}^{2}}{\sigma^{5}}
\end{aligned}
$$

Now when $H(\sigma, \lambda)=\sigma$, then

$$
w_{1}=1, \quad w_{2}=0, \quad w_{i j}=0, \quad \text { for } i, j=1,2 .
$$

Therefore,

$$
\begin{gathered}
A=0, \quad A_{12}=\tau_{11}, \quad A_{21}=\tau_{12} \quad B_{12}=\tau_{11}^{2}, \\
B_{21}=\tau_{21} \tau_{22}, \quad C_{12}=3 \tau_{11} \tau_{12}, \quad C_{21}=\tau_{22} \tau_{11}+2 \tau_{21}^{2} .
\end{gathered}
$$


Finally, using $p=\log \pi(\sigma, \lambda)$, we obtain

$$
p_{1}=\frac{\lambda \delta+b}{\sigma^{2}}-\frac{a+v+1}{\sigma} \quad \text { and } \quad p_{2}=\frac{v-1}{\lambda}-\frac{\delta}{\sigma} .
$$

Similarly, when $H(\sigma, \lambda)=\lambda$, then

$$
w_{1}=0, \quad w_{2}=1, \quad w_{i j}=0, \quad \text { for } i, j=1,2 .
$$

Therefore,

$$
\begin{array}{rlrl}
A & =0, & A_{12}=\tau_{21}, \quad A_{21}=\tau_{22}, & B_{12}=\tau_{12} \tau_{11}, \\
B_{21} & =\tau_{22}^{2}, \quad C_{12}=\tau_{11} \tau_{22}+2 \tau_{12}^{2}, & C_{21}=3 \tau_{22} \tau_{21} .
\end{array}
$$

\section{References}

Ahmadi, J. and Balakrishnan, N. (2011). Distribution-free prediction intervals for order statistics based on record coverage, Journal of the Korean Statistical Society, 40, 181-192.

Balakrishnan, N., Ahsanullah, M. and Chan, P. S. (1992). Relations for single and product moments of record values from Gumbel distribution, Statistical and Probability Letters, 15, 223-227.

Chandler, K. N. (1952). The distribution and frequency of record values, Journal of the Royal Statistical Society, Series B, 14, 220-228.

Howlader, H. A. and Hossain, A. M. (2002). Bayesian Survival estimation of Pareto distribution of the second kind based on failure-censored data, Computational Statistics and Data Analysis, 38, 301-314.

Jaheen, Z. F. (2003). A Bayesian analysis of record statistics from the Gompertz model, Applied Mathematics and Computation, 145, 307-320.

Kang, S. B., Cho, Y. S. and Han, J. T. (2009). Estimation for the half logistic distribution based on double hybrid censored samples, Communications of the Korean Statistical Society, 16, 10551066.

Kang, S. B. and Seo, J. I. (2011). Estimation in an exponentiated half logistic distribution under progressively type-II censoring, Communications of the Korean Statistical Society, 18, 657-666.

Kim, Y. K., Kang, S. B. and Seo, J. I. (2011a). Bayesian estimations on the exponentiated half triangle distribution under type-I hybrid censoring, Journal of the Korean Data \& Information Science Society, 22, 565-574.

Kim, Y. K., Kang, S. B. and Seo, J. I. (2011b). Bayesian estimations on the exponentiated distribution family with type-II right censoring, Communications of the Korean Statistical Society, 18, 603613.

Kundu, D. and Gupta, R. D. (2005). Estimation of $P[Y>X]$ for generalied exponentioal distribution, Metrika, 61, 291-308.

Lindley, D. V. (1980). Approximated Bayes method, Trabajos de Estadistica, 31, 223-237.

Nadarajah, S. (2006). The exponentiated Gumbel distribution with climate application, Environmetrics, 17, 13-23.

Soliman, A. A., Abd Ellah, A. H. and Sultan, K. S. (2006). Comparison of estimates using record statistics from Weibull model: Bayesian and non-Bayesian approaches, Computational Statistics \& Data Analysis, 51, 2065-2077.

Varian, H. R. (1975). A Bayesian approach to real estate assessment. In: S. E. Feinberg and A. Zellner, Eds., Studies in Bayesian Econometrics and Statistics in Honor of Leonard J. Savage, North Holland, Amsterdam, 195-208. 
Zellner, A. (1986). Bayesian estimation and prediction using asymmetric loss function, Journal of American Statistical Association, 81, 446-451.

Received July 11, 2013; Revised September 12, 2013; Accepted September 12, 2013 